\title{
Implicações clínicas orais e a importância dos cuidados odontológicos em pacientes sob cuidados paliativos: Revisão integrativa da literatura
}

\author{
Oral clinical implications and the importance of dental care in palliative care patients: An \\ integrative literature review \\ Implicaciones clínicas orales y la importancia del cuidado dental en pacientes en cuidados \\ paliativos: Revisión bibliográfica integradora
}

Recebido: 17/07/2021 | Revisado: 24/07/2021 | Aceito: 25/07/2021 | Publicado: 01/08/2021

\author{
Luísa Carolina Santos Lima \\ ORCID: https://orcid.org/0000-0002-2284-2968 \\ Centro Universitário de Patos de Minas, Brasil \\ E-mail: luisacs1@unipam.edu.br \\ Mariana Martins Andalécio \\ ORCID: https://orcid.org/0000-0002-7722-8199 \\ Centro Universitário de Patos de Minas, Brasil \\ E-mail: marianamartins@unipam.edu.br \\ Rodrigo Soares de Andrade \\ ORCID: https://orcid.org/0000-0001-6114-0929 \\ Centro Universitário de Patos de Minas, Brasil \\ E-mail: rodrigosa@unipam.edu.br \\ Fabrício Campos Machado \\ ORCID: https://orcid.org/0000-0003-4603-8795 \\ Centro Universitário de Patos de Minas, Brasil \\ E-mail: fabriciocampos@unipam.edu.br \\ Veridiana Resende Novais \\ ORCID: https://orcid.org/0000-0001-9017-2946 \\ Universidade Federal de Uberlândia, Brasil \\ E-mail: veridiana.novais@ufu.br \\ Thiago de Amorim Carvalho \\ ORCID: https://orcid.org/0000-0003-1153-0931 \\ Centro Universitário de Patos de Minas, Brasil \\ E-mail: thiagocarvalho@unipam.edu.br
}

\begin{abstract}
Resumo
O objetivo deste artigo é caracterizar as implicações clínicas orais e a potencial contribuição da Odontologia para a qualidade de vida de pessoas em cuidados paliativos. Foram pesquisados artigos dos últimos 10 anos, relacionados ao assunto nas bases de dados PUBMED e Scielo com as palavras-chave: "cuidados paliativos", "cuidado dental" e "saúde bucal" e no inglês "palliative care", "dental care" ou "oral health". Foram selecionados 7 artigos a partir da busca sistematizada e com a aplicação dos critérios de inclusão e exclusão. As principais manifestações orais de pacientes em cuidados paliativos são a xerostomia, a cárie dental, a candidose e a mucosite, e a presença do cirurgião dentista parece contribuir para a manutenção da qualidade de vida em níveis aceitáveis, ainda que as pessoas estejam em processo de morte. Percebe-se pelo exposto que a abordagem do cirurgião dentista é necessária no âmbito da promoção de saúde, prevenção de agravos e recuperação de reabilitação de danos, que possibilitam a manutenção da qualidade de vida e que esta atenção ainda é negligenciada, devido a falta de obrigatoriedade do cirurgião dentista nas linhas de cuidado de pacientes terminais. Mais estudos sobre a influência dos cuidados odontológicos na manutenção da qualidade de vida em pacientes sob cuidados paliativos são necessários para fomentar e ratificar a importância do cirurgião dentista na equipe de cuidados integrais em saúde desse público.
\end{abstract}

Palavras-chave: Odontologia; Cuidados paliativos; Qualidade de vida.

\begin{abstract}
The aim of this article is to characterize the oral clinical implications and the potential contribution of dentistry to the quality of life of people in palliative care. Articles from the last 10 years, related to the subject were searched in the PUBMED and Scielo databases with the keywords: "palliative care", "dental care" and "oral health". Seven articles were selected from the systematized search and with the application of inclusion and exclusion criteria. The main oral manifestations of patients in palliative care are xerostomy, dental caries, candidosis and mucositis, and the presence of the dental surgeon seems to contribute to the maintenance of quality of life at acceptable levels, even though people
\end{abstract}


are dying. It is perceived by the exposed that the approach of the dental surgeon is necessary in the scope of health promotion, prevention of aggravations and recovery of damage rehabilitation, which make possible the maintenance of quality of life and that this attention is still neglected, due to the lack of obligation of the dental surgeon in the lines of care of terminal patients. More studies on the influence of dental care in the maintenance of quality of life in patients under palliative care are necessary to promote and ratify the importance of the dental surgeon in the team of integral health care of this public.

Keywords: Dentistry; Palliative care; Quality of life.

\section{Resumen}

El objetivo de este artículo es caracterizar las implicaciones clínicas orales y la posible contribución de la odontología a la calidad de vida de las personas en cuidados paliativos. Se buscaron artículos de los últimos 10 años, relacionados con el tema, en las bases de datos PUBMED y Scielo con las palabras clave: "palliative care", "dental care" y "oral health". Se seleccionaron siete artículos a partir de la búsqueda sistematizada y con la aplicación de los criterios de inclusión y exclusión. Las principales manifestaciones orales de los pacientes en cuidados paliativos son la xerostomía, la caries dental, la candidosis y la mucositis, y la presencia del cirujano dentista parece contribuir al mantenimiento de la calidad de vida en niveles aceptables, aunque las personas estén en proceso de muerte. Se percibe por la exposición que el abordaje de la cirugía dental es necesario en el ámbito de la promoción de la salud, la prevención de agravios y la recuperación y rehabilitación de los daños, que posibilitan la manutención de la calidad de vida y que esta atención aún es negligente, debido a la falta de obrigación de la cirugía dental en las líneas de cuidado de los pacientes terminales. Son necesarios más estudios sobre la influencia de la atención odontológica en el mantenimiento de la calidad de vida de los pacientes en cuidados paliativos para promover y ratificar la importancia del cirujano dentista en el equipo de atención integral de la salud de este público.

Palabras clave: Odontología; Cuidados paliativos; Calidad de vida.

\section{Introdução}

A Organização Mundial da Saúde (OMS, 2002) definiu cuidados paliativos (CP) como uma estratégia de promoção melhoria da qualidade de vida a pacientes e familiares que são acometidos por doenças que interrompam a sequência da vida, sanando a dor e sofrimento. Para tal fim, se faz necessário a identificação, avaliação e tratamento precoce da dor e de outros contratempos de origens diversas que possam permear esse momento (Miranda, 2016). Este conceito veio sofrendo modificações durante as décadas, até que em 2017 a OMS atualizou o termo ao afirmar que os cuidados paliativos se tratam de condutas transversais nas etapas do cuidado, associadas às condições terminais e limitadoras da vida, como também de doenças crônicas progressivas (Tritany, Souza Filho \& Mendonça, 2021).

A Odontologia é uma área com vários campos de atuação, que tem o intuito de promover e reestabelecer o bem-estar e a saúde dos indivíduos. Muitas vezes, o cirurgião dentista atua em cuidados paliativos para minimizar o sofrimento e desconforto, precavendo a aparição de novos problemas, especialmente os orais, em pacientes que se encontram em estado terminal. Aos enfermos é indispensável a atuação de uma equipe multiprofissional para assegurar atendimento qualificado e seguro ao paciente. O cirurgião-dentista é inserido nessa linha de cuidado para garantir assistência integral e integrada aos internados, e irá atuar detectando alterações na região da cabeça e do pescoço (Macedo et al., 2020; Negi et al., 2016). Trabalhos em outras áreas do conhecimento como a enfermagem e a fonoaudiologia são encontrados na literatura, inclusive com o escopo desta revisão de demonstrar os benefícios que a assistência destas áreas do conhecimento apresenta quando ofertada a pessoas em cuidados paliativos, mas em Odontologia trabalhos com esse foco são escassos (Silveira, Costa, Lohmann \& Lavall, 2020; Pacheco et al., 2020; Costa \& Silva, 2021)

Uma análise detalhada, baseada no comprometimento sistêmico se faz imperiosa para prevenção, promoção e diminuição de complicações orais (Mulk et al., 2014), uma vez que devido ao estado do paciente ele apresenta-se predisposto as alterações. Esses transtornos são causados por uma série de fatores como o uso frequente, intenso e duradouro de medicamentos, a diminuição salivar, a permanência da cavidade oral fechada e muitas vezes o uso de tubos orotraqueais. Levase em consideração o fato de a boca ser um espaço com grande potencial patogênico devido à presença de grande quantidade de microrganismos, o que predispõe a distúrbios sistêmicos, tornando-se um fator de degradação durante a internação 
hospitalar (Austriaco-Leite et al., 2018). Os hábitos de higiene são constantemente negligenciados neste meio, embora seja, importante para a promoção de saúde dos que ali estão (Souto, Santos \& Cavalcante, 2019).

O principal foco do dentista na atuação do cuidado paliativo deve ser eliminar focos inflamatórios, infecciosos e dores decorrente das questões bucais, a partir de planejamentos éticos, humanos e interdisciplinares. Observa-se a frequente manifestação de acúmulo de biofilme, doença periodontal (gengivite e periodontite), doenças oportunistas (candidose), lesões de cárie, mucosite, xerostomia entre outras alterações resultantes da imunossupressão, diminuição do fluxo salivar e uso excessivo de medicamentos (Miranda, 2016; Crispim, Gonçalo \& Pereira, 2020). Intervenções básicas como a adequação mecânica, eliminação de saburra lingual e aplicação de soluções que diminuem a colonização bacteriana, quando executadas de maneira regular e satisfatória, podem influenciar diretamente no prognóstico do paciente. O direcionamento de conduta dos enfermeiros e técnicos de enfermagem sobre ações preventivas direcionadas ao sistema estomatognático deve ocorrer para que se obtenha o cuidado adequado da região (Miranda \& Olivia 2015).

Infelizmente o Projeto de Lei da Câmara $\mathrm{n}^{\circ}$ 34, de 2013, que tornava obrigatória a prestação de assistência odontológica a pacientes em regime de internação hospitalar, aos portadores de doenças crônicas e, ainda, aos atendidos em regime domiciliar, foi vetado em 2019 pelo atual presidente da república que justificou o veto com medidas econômicas, já que em médio e longo prazo a vigência da lei promoveria grandes impactos aos cofres públicos (CFO, 2019).

O objetivo deste trabalho é caracterizar as implicações clínicas orais e a potencial contribuição da Odontologia para a qualidade de vida de pessoas em cuidados paliativos.

\section{Metodologia}

O presente estudo trata-se de uma revisão integrativa de literatura de abordagem exploratória e qualitativa. A pergunta norteadora para delineamento temático, elaborada por meio da estratégia PICO foi: qual a atuação do cirurgião dentista e as potenciais contribuições da assistência odontológica para pessoas em cuidados paliativos? Para o desenvolvimento deste estudo, foi realizada uma busca nas bases de dados eletrônicas PUBMED e Scielo, baseada nos desfechos de principais manifestações orais em pessoas sob cuidados paliativos, as intervenções mais realizadas pelo cirurgião dentista nesse público e as eventuais melhorias nas condições sistêmicas e na qualidade de vida de pessoas sob cuidados paliativos, após o estabelecimento de um plano de cuidados odontológicos. Para as buscas foram utilizados os descritores "cuidados paliativos", "cuidado dental" e "saúde bucal" e no inglês "palliative care", "dental care" e "oral health" associados por meio dos operadores booleanos "e" e "ou" e seus correspondentes em inglês. A partir da leitura dos artigos encontrados primariamente, foram selecionados os que tinham relação com o objetivo e os desfechos, a partir do título, inicialmente, seguida da leitura do resumo e aqueles que foram selecionados foram lidos na íntegra para análise do conteúdo.

Os critérios de inclusão dos artigos foram redação em português ou inglês, artigos disponíveis na íntegra, publicados nos últimos 10 anos, e artigos dos tipos revisões de literatura, revisões sistemáticas da literatura, revisões sistemáticas com metanálise e ensaios clínicos randomizados. Foram excluídos da análise qualitativas os artigos publicados há mais de 10 anos, publicações em idioma diverso ao estabelecido, aqueles nos quais não era possível a localização do texto completo, opiniões de especialistas, e a literatura cinzenta que consta de teses, dissertações, monografias, trabalhos de conclusão de curso e capítulos de livro.

\section{Resultados}

A Figura 1 demonstra o processo de seleção dos trabalhos por meio das chaves de busca e da aplicação sistematizada dos critérios de inclusão e exclusão citados na metodologia. O fluxograma leva em consideração os critérios elencados pela estratégia PRISMA para elegibilidade de artigos a serem discutidos em revisões sistemáticas e de escopo (Page et al., 2021). 
Figura 1 - Fluxograma do processo de seleção dos estudos primários adaptado do Preferred Reporting Items for Systematic review and Meta-Analyses (PRISMA).

Identificação

Filtro: 10 anos

Textos completos
Registro identificado no banco de dados de busca (Pubmed -

Palliative care and dental care $\mathrm{n}=64$

Palliative care and oral health $\mathrm{n}=171$ )

Registro identificado no banco de dados de busca (Scielo -

Palliative care and dental care $\mathrm{n}=4$

Palliative care and oral health $\mathrm{n}=128$ )

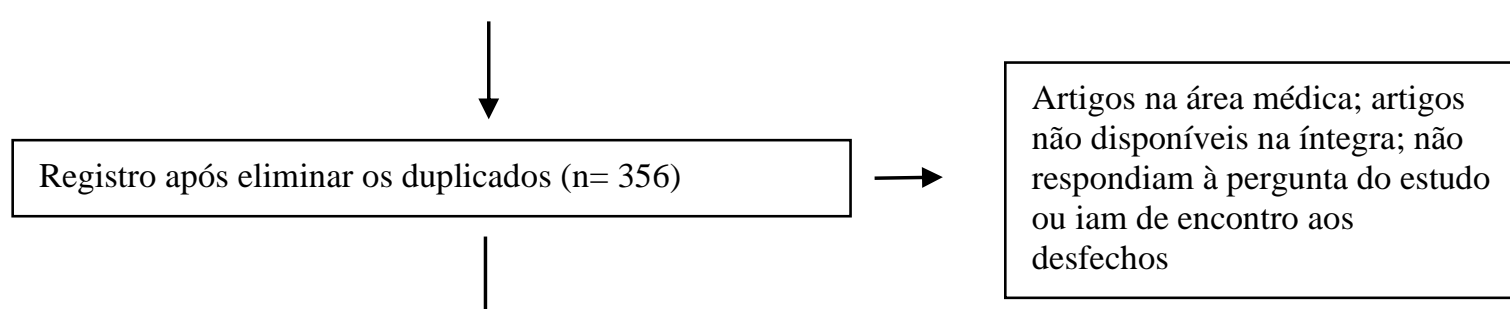

Seleção

Registros selecionados

Registros excluídos após a leitura

dos resumos $(\mathrm{n}=11)$

Inclusão

Estudos incluídos em síntese qualitativa $(\mathrm{n}=7)$

Fonte: Autores.

Após a seleção dos 7 artigos para a análise de conteúdo, estes foram caracterizados por nome do autor e ano de publicação, o periódico no qual o artigo foi publicado, o objetivo do trabalho, o tipo de estudo e os principais resultados. Tal caracterização esta disponível no Quadro 1. 
Quadro 1 - Caracterização dos estudos selecionados para análise qualitativa.

\begin{tabular}{|c|c|c|c|c|}
\hline $\begin{array}{l}\text { Autor e ano de } \\
\text { publicação }\end{array}$ & Periódico & Objetivo & $\begin{array}{l}\text { Tipo de } \\
\text { Estudos }\end{array}$ & Resultados \\
\hline $\begin{array}{l}\text { Dhaliwal et al., } \\
2020\end{array}$ & $\underline{\underline{\text { Indian J Palliat }}} \underline{\underline{\text { Care. }}}$ & $\begin{array}{l}\text { Identificar os tipos de microflora na } \\
\text { cavidade oral de pacientes paliativos; } \\
\text { Identificar as técnicas utilizadas para } \\
\text { investigar a presença microbiológica na } \\
\text { cavidade oral de pacientes paliativos. }\end{array}$ & $\begin{array}{l}\text { Revisão } \\
\text { integrativa } \\
\text { da literatura }\end{array}$ & $\begin{array}{l}\text { Encontrou Candida como a microflora } \\
\text { mais comum na cavidade oral de } \\
\text { pacientes paliativos, com C. albicans } \\
\text { sendo a espécie predominante. }\end{array}$ \\
\hline $\begin{array}{l}\text { Fitzagerald \& } \\
\text { Gallangher, } \\
2018\end{array}$ & $\begin{array}{l}\text { Dentist Spac } \\
\text { Care }\end{array}$ & $\begin{array}{l}\text { Sintetizar evidências existentes na } \\
\text { cavidade oral de pacientes sob cuidados } \\
\text { paliativos, para ajudar médicos e } \\
\text { tomadores de decisões, a aumentarem a } \\
\text { conscientização dos desafios para esses } \\
\text { pacientes, e facilitar pesquisas futuras. }\end{array}$ & $\begin{array}{l}\text { Revisão de } \\
\text { literatura }\end{array}$ & $\begin{array}{l}\text { Há uma escassez de pesquisas } \\
\text { relacionadas ao apoio odontológico a } \\
\text { pacientes sob cuidados paliativos e a } \\
\text { necessidade de enfatizar os cuidados } \\
\text { diários e lidar com as limitações do } \\
\text { cuidado pessoal. }\end{array}$ \\
\hline $\begin{array}{l}\text { Kvalheim et al., } \\
2019\end{array}$ & Eur. J. Oral Sci. & $\begin{array}{l}\text { Comparar a eficácia de três hidratantes } \\
\text { orais usados em pacientes paliativos } \\
\text { para o controle da xerostomia. }\end{array}$ & $\begin{array}{l}\text { Estudo de } \\
\text { caso contole }\end{array}$ & $\begin{array}{l}\text { Nenhum dos três produtos teste usados } \\
\text { no tratamento da xerostomia foram } \\
\text { considerados apropriados clinicamente } \\
\text { para uso. }\end{array}$ \\
\hline $\begin{array}{l}\text { Mulk et al., } \\
2014\end{array}$ & $\begin{array}{l}\text { J Clin Diagn } \\
\underline{\text { Res }}\end{array}$ & $\begin{array}{l}\text { Descrever o papel do dentista na equipe } \\
\text { paliativa. }\end{array}$ & $\begin{array}{l}\text { Revisão de } \\
\text { literatura }\end{array}$ & $\begin{array}{l}\text { Pacientes em estágio terminal } \\
\text { necessitam de atenção especializada } \\
\text { multiprofissional e os dentistas devem } \\
\text { ser incluídos nessa equipe, uma vez que } \\
\text { a saúde oral é frequentemente } \\
\text { negligenciada. }\end{array}$ \\
\hline Sen et al., 2020 & $\begin{array}{l}\text { J. Family Med } \\
\text { Prim Care. }\end{array}$ & $\begin{array}{l}\text { A importância da atuação do cirurgião- } \\
\text { dentista na melhoria da qualidade de } \\
\text { vida desses pacientes. }\end{array}$ & $\begin{array}{l}\text { Revisão } \\
\text { integrativa } \\
\text { da literatura }\end{array}$ & $\begin{array}{l}\text { O papel do cirurgião dentista como } \\
\text { atenção primária deve ser antecipado e } \\
\text { sempre incluído na unidade de cuidados } \\
\text { paliativos para a melhoria do paciente. }\end{array}$ \\
\hline $\begin{array}{l}\text { Souto, Santos \& } \\
\text { Cavalcante, } \\
2019\end{array}$ & $\begin{array}{l}\text { RGO, Rev. } \\
\text { Gaúch. Odontol. }\end{array}$ & $\begin{array}{l}\text { Apresentar a importância da atenção } \\
\text { odontológica voltada para pacientes } \\
\text { com câncer sob cuidados paliativos }\end{array}$ & $\begin{array}{l}\text { Revisão de } \\
\text { literatura }\end{array}$ & $\begin{array}{l}\text { Pacientes oncológicos em cuidados } \\
\text { paliativos devem ter junto a equipe } \\
\text { multiprofissional o apoio de um } \\
\text { cirurgião dentista, focado em promover } \\
\text { qualidade de vida. }\end{array}$ \\
\hline $\begin{array}{l}\text { Venkatasalu et } \\
\text { al., } 2020\end{array}$ & $\begin{array}{l}\text { BMC Oral } \\
\text { Health }\end{array}$ & $\begin{array}{l}\text { Sintetizar as evidências publicadas, } \\
\text { sobre as condições orais entre pacientes } \\
\text { paliativos, impacto, gestão e desafios } \\
\text { nas condições bucais entre pacientes } \\
\text { paliativos. }\end{array}$ & $\begin{array}{l}\text { Revisão } \\
\text { integrativa } \\
\text { da literatura }\end{array}$ & $\begin{array}{l}\text { São diversas as condições orais que } \\
\text { comprometem a cavidade bucal de } \\
\text { pacientes paliativos e estão surgindo } \\
\text { diferentes tipos de tratamento para o } \\
\text { manejo destas condições, fornecendo } \\
\text { assim, conhecimento básico de } \\
\text { orientação a profissionais da saúde para } \\
\text { promover qualidade de vida. }\end{array}$ \\
\hline
\end{tabular}

Fonte: Autores.

Foram analisados 6(seis) revisões de literatura e 1(um) estudo epidemiológico do tipo caso controle que abordam a temática e dentre estes, pode-se observar que os enfermos que necessitam deste cuidado possuem em sua maioria complicações sistêmicas. A presença de neoplasias malignas, a necessidade de quimioterapia e radioterapia, pacientes geriátricos e em estado terminal decorrente de outras comorbidades, foram os que demonstraram maior necessidade da prestação de cuidados paliativos.

\section{Discussão}

As manifestações orais mais prevalentes foram xerostomia, candidíase, cárie dental e mucosite. Foi relatado também a presença de dor orofacial, alterações nutricionais e de paladar, raízes residuais, úlceras, disfagia, osteorradionecrose, doenças periodontais e próteses mal adaptadas.

A xerostomia está diretamente ligada aos cuidados paliativos devido à prevalência nestes pacientes de neoplasias em 
regiões de cabeça e pescoço que carecem de tratamentos com radiação, ou em outros sítios do organismo que podem requerer tratamento quimioterápico que pode ocasionar muitos efeitos adversos. No caso de radioterapia em região de cabeça e pescoço, o mecanismo da hipossalivação e consequente xerostomia, ocorre já que o componente parenquimatoso das glândulas salivares é altamente sensível a radiação, a glândula torna-se fibrótica, reduzindo a microcirculação e ocorre uma degradação parenquimatosa, diminuindo então, o fluxo salivar (Sen, et. al 2020). A xerostomia, ou sensação de boca seca, tem uma prevalência de 50 a 92\%, nos pacientes em cuidados paliativos de acordo com os estudos revisados.

Com a saliva mais espessa e com menor capacidade de lubrificação, o pH salivar diminui, predispondo assim os pacientes à maior incidência de lesões de cárie. Essa maior atividade de cárie pode perdurar pelos três primeiros meses do tratamento radioterápico e tende a diminuir do $6^{\circ}$ ao $12^{\circ}$ mês, com a instituição de protocolos de reposição salivar e de higiene bucal rigorosa que culmina com o melhor controle da placa. Além disso o uso do flúor seja no veículo aquoso, em gel ou verniz, pode contribuir para um melhor controle da atividade de cárie (Fitzgerald, et. al 2018).

Kvalheim et al. (2019) demonstra três alternativas de hidratantes orais para o tratamento da xerostomia, que consiste em $17 \%$ de solução aquosa de glicerol, composto oxigenado de glicerol e o substituto salivar Salient ${ }^{\circledR}($ Salient Pharma, Dinamarca). Destes produtos o glicerol foi o que promoveu alívio imediato da xerostomia, mas após 2 horas, não apresentou tal eficácia, levando a conclusão de que nenhum dos produtos é clinicamente suficiente no controle da xerostomia, já que esta é multifatorial.

A respeito da candidose, deve considerar que a cavidade oral abriga uma infinidade de microrganismos, inclusive a Candida albicans, agente etiológico dessa condição, que se trata de um fungo residente. Quando ocorre o desequilíbrio da microflora oral, ou uma queda importante da imunidade do paciente, pode-se observar uma proliferação generalizada desse fungo, que pode acometer a condição bucal, evoluindo em quadros mais graves para um comprometimento sistêmico do paciente (Dhaliwal et al., 2020). Estima-se que a prevalência da candidíase em pessoas sob cuidados paliativos por tratamento antineoplásico, é de 70 a $85 \%$ e ocorre devido a quimioterapia, radioterapia e principalmente como consequência da xerostomia (Souto, Santos \& Cavalcante, 2019; Mulk et al., 2014).

Como tratamento para esta condição, pode-se optar pela aplicação tópica ou sistêmica de medicamentos, além da rigorosa instrução de higiene oral. Alguns fármacos que podem ser administrados são clotrimazol e a nistatina (topicamente) e o cetoconazol e fluconazol em casos que a terapêutica tópica não apresentou resultados satisfatórios (Dhaliwal et al., 2020).

Por fim, tem-se a mucosite que se manifesta como uma úlcera dolorosa, que acomete regiões como o revestimento da mucosa oral, palato mole e faringe. O principal fator causal é a quimioterapia, mas essa alteração também pode ser consequência da radioterapia. Essa alteração patológica varia entre 4 classificações que vão desde formas brandas que provocam úlceras dolorosas, edema e leve dor de garganta, sem, no entanto, comprometer a alimentação via oral, até o estágio 4 no qual é necessário suporte alimentar parenteral ou entérico devido a estomatite grave. Para prevenção da mucosite após a quimioterapia e radioterapia, administra-se amifostina, que irá remover os radicais livres do tecido que são responsáveis por reforçar os efeitos da mucosite. Outra medida profilática é o uso do LASER de baixa intensidade, antes, durante e depois das sessões de quimioterapia (Souto, Santos \& Cavalcante, 2019).

Venkatasalu et. al (2020) demonstram em seu trabalho que $40 \%$ dos pacientes que são acometidos por doenças que necessitam de cuidados paliativos não se apresentam aptos para comunicar os problemas orais presentes e não efetua a higiene oral necessária corretamente sem assistência, o que acaba gerando o agravamento das doenças e quadros de dor e desconforto. Estas ocasiões podem ser evitadas quando se tem o acompanhamento regular do cirurgião dentista direcionado para esse tipo de paciente, uma vez que este pode realizar o diagnóstico precoce, evitando assim a piora do quadro clínico dos doentes.

Fitzgerald e Gallagher (2018) trazem em seu estudo que a qualidade de vida e a saúde oral estão diretamente relacionadas uma vez que os cuidados orais subsidiam melhorias no quadro dos pacientes paliativos. O profissional da área vai 
atuar no controle dos riscos de infecções que são potencializadas com a presença do biofilme, o controle e acompanhamento da aparição de alterações patológicas que podem agravar o quadro clínico pré-existente.

Apesar de comprovada a eficácia da atuação do cirurgião dentista, ainda possui muitos obstáculos para que seu trabalho seja realizado de maneira efetiva, uma vez que sua presença não é obrigatória nos ambientes de cuidado onde o grupo de pacientes abordados no estudo se encontram. Sendo assim os procedimentos de higiene oral são realizados por profissionais da enfermagem, que não possuem formação específica para os cuidados orais como os cirurgiões dentistas, para realizar esta atividade. Alguns dos obstáculos encontrados na rotina diária são, a não obrigatoriedade do cirurgião dentista nas equipes multiprofissionais de cuidados domiciliares ou hospitalares em pacientes sob cuidados paliativos, o que acarreta falta de treinamento adequado da equipe para fins de cuidados orais, o manejo e cooperação do paciente, recursos disponíveis para o atendimento e o acesso à cavidade oral.

\section{Conclusão}

Após o exposto percebe-se que as principais condições orais presentes em pacientes paliativos que consistem em xerostomia, candidíase, cárie dental e mucosite que ora podem ser causadas por queda de imunidade, ou por consequências de tratamento para as doenças de base que culminam na inserção do paciente em programas de cuidados paliativos, como o tratamento antineoplásico. A abordagem do cirurgião dentista é necessária no âmbito da promoção de saúde, prevenção de agravos e recuperação de reabilitação de danos, que possibilitam a manutenção da qualidade de vida, ainda que a pessoa já se encontre em processo de morte. Esta atenção ainda é negligenciada, devido a falta de obrigatoriedade do cirurgião dentista nas linhas de cuidado de pacientes terminais. Mais estudos sobre a influência dos cuidados odontológicos na manutenção da qualidade de vida em pacientes sob cuidados paliativos são necessários para fomentar e ratificar a importância do cirurgião dentista na equipe de cuidados integrais, além de fornecer informações baseadas em evidência científica robusta sobre a propedêutica clínica a ser instituída para esse público.

\section{Referências}

Austríaco-Leite, H. L., Ferreira-Lopes, F., Alves Cardoso, M. do S., \& Diniz-Souza, L. C. (2018). Avaliação odontológica de pacientes em unidade de terapia intensiva (UTI) pediátrica. CES Odontología, 31(2), 6-14. https://doi.org/10.21615/cesodon.31.2.1

Bastos, P.L., Mesquita, T.C., Ottoboni, G.S., Figueiredo, V.M.G. (2015). Métodos de higienização em próteses dentais removíveis. Revista Bahiana de Odontologia. Ago;6(2):129-137. http://dx.doi.org/10.17267/2596-3368dentistry.v6i2.683

Page, M. J., Moher, D., Bossuyt, P. M., Boutron, I., Hoffmann, T. C., Mulrow, C. D., Shamseer, L., Tetzlaff, J. M., Akl, E. A., Brennan, S. E., Chou, R., Glanville, J., Grimshaw, J. M., Hróbjartsson, A., Lalu, M. M., Li, T., Loder, E. W., Mayo-Wilson, E., McDonald, S., McGuinness, L. A., \& McKenzie, J. E. (2021). PRISMA 2020 explanation and elaboration: updated guidance and exemplars for reporting systematic reviews. BMJ (Clinical research ed.), 372, n160. https://doi.org/10.1136/bmj.n160

Calazans, M. (2019). Bolsonaro veta projeto que garante assistência odontológica a pacientes em regime de internação hospitalar. CFO. https://website.cfo.org.br/bolsonaro-veta-projeto-que-garante-assistencia-odontologica-a-pacientes-em-regime-de-internacao-hospitalar/.

Costa, B. M., \& Silva, D. A. da. (2021). Atuação da equipe de enfermagem em cuidados paliativos. Research, Society and Development, $10(2)$, e28010212553. https://doi.org/10.33448/rsd-v10i2.12553

Crispim, D. H., Gonçalo, T. C. A. \& Pereira, E. A. L. (2020). Remuneração em cuidados paliativos 2020. Academia Nacional de cuidados paliativos. Academia Nacional de Cuidados Paliativos. ISBN 978-65-990595-6-8

Damascena, L. C. L., Rodrigues, L. V., Costa, R. C., Da Nóbrega, J. B. M., Dantas, E. L. A. \& Valença, A. M. G. (2017). Factors associated with oral biofilm in ICU patients with infectious diseases. Revista de Odontologia da UNESP 46(6), 343-350. <https://doi.org/10.1590/18072577.05117https://doi.org/10.1590/1807-2577.05117.

Dhaliwal, J. S., Murang, Z. R., Ramasamy, D., \& Venkatasalu, M. R. (2020). Oral Microbiological Evidence among Palliative Patients: An Integrated Systematic Review. Indian journal of palliative care, 26(1), 110-115. https://doi.org/10.4103/IJPC.IJPC_178_19

Fitzgerald, R., \& Gallagher, J. (2018). Oral health in end-of-life patients: A rapid review. Special care in dentistry: official publication of the American Association of Hospital Dentists, the Academy of Dentistry for the Handicapped, and the American Society for Geriatric Dentistry, 38(5), $291-298$. https://doi.org/10.1111/scd.12316 
Research, Society and Development, v. 10, n. 9, e52410918356, 2021

(CC BY 4.0) | ISSN 2525-3409 | DOI: http://dx.doi.org/10.33448/rsd-v10i9.18356

Kvalheim, S. F., Marthinussen, M. C., Haugen, D. F., Berg, E., Strand, G. V., \& Lie, S. A. (2019). Randomized controlled trial of the effectiveness of three different oral moisturizers in palliative care patients. European journal of oral sciences, 127(6), 523-530. https://doi.org/10.1111/eos.12655

Macedo, M.P., Souza, L.C.D., Corrêa, R. Da G.C.F. \& Lopes, F.F. (2020). Aspectos do atendimento odontológico a pacientes internados em uma clínica médica de um hospital universitário. [Aspects of dental care for patients hospitalized in a medical clinic from a university hospital] ABCS Health Sciences. 45, 1198, 22 de outubro de 2020. https://doi.org/10.7322/abcshs.45.2020.1198

Meinberg, M. C., Cheade, M., Miranda, A. L., Fachini, M. M., \& Lobo, S. M. (2012). The use of $2 \%$ chlorhexidine gel and toothbrushing for oral hygiene of patients receiving mechanical ventilation: effects on ventilator-associated pneumonia. Revista Brasileira de terapia intensiva, 24(4), 369-374. https://doi.org/10.1590/s0103-507x2012000400013

Miranda, A. F., Oliva, A. (2015). Cuidados Paliativos e Odontogeriatria: Breve Comunicação. REVISTA PORTAL de Divulgação, 44(5) 2178-3454.

Miranda, A.F. (2016). Oral Health and Care at Intensive Care Units; Journal of Nursing and Care. 5(375). Catholic University of Brasília, Brasil (HCBr), Brasília, Brazil. 10.4172/2167-1168.1000375.

Mulk, B. S., Chintamaneni, R. L., Mpv, P., Gummadapu, S., \& Salvadhi, S. S. (2014). Palliative dental care- a boon for debilitating. Journal of Clinical and Diagnostic Research: JCDR, 8(6), ZE01-ZE6. https://doi.org/10.7860/JCDR/2014/8898.4427

Negi, S.B., Anjana, C.M., Anita B., Nileena R.K., Haris P.S. (2016). O papel dos dentistas nos cuidados paliativos. Revista IOSR de Odontologia e Ciências Médicas (IOSR-JDMS). 01-04. 10.9790 / 0853-1504040104.

Pacheco, L. da S. P., Santos, G. S. dos, Machado, R., Granadeiro, D. da S., Melo, N. G. S. de, \& Passos, J. P. (2020). O processo de comunicação eficaz do enfermeiro com o paciente em cuidados paliativos. Research, Society and Development, 9(8), e747986524. https://doi.org/10.33448/rsd-v9i8.6524

Sen, S., Priyadarshini, S. R., Sahoo, P. K., Dutta, A., Singh, A. K., \& Kumar, U. (2020). Palliative oral care in patients undergoing radiotherapy: Integrated review. Journal of Family Medicine and Primary Care, 9(10), 5127-5131. https://doi.org/10.4103/jfmpc.jfmpc_827_20

Silveira, P. J. da, Costa, A. E. K. da, Lohmann, P. M., \& Lavall, E. (2020). Revisão integrativa: cuidados paliativos em pacientes oncológicos. Research, Society and Development, 9(2), e144922136. https://doi.org/10.33448/rsd-v9i2.2136

Souto, K. C. L., Santos, D. B. N. \& Cavalcanti, U. D. N. T. (2019). Dental care to the oncological patient in terminality. RGO - Revista Gaúcha de Odontologia [online]. 67, e20190032. <https://doi.org/10.1590/1981-86372019000323592>.

Tritany, E. F., Souza Filho, B. A. B. \& Mendonça, P. E. X. (2021). Fortalecer os Cuidados Paliativos durante a pandemia de Covid-19. Interface (Botucatu) [online]. 25(1), e200397. https://doi.org/10.1590/interface.200397.

Venkatasalu, M. R., Murang, Z. R., Ramasamy, D. T. R. \& Dhaliwal, J. S. (2020). Oral health problems among palliative and terminally ill patients: an integrated systematic review. BMC Oral Health 20(79). https://doi.org/10.1186/s12903-020-01075-w 